\title{
La médecine constitutive de la nouvelle science de l'homme : Cabanis
}

\section{Mariana Saad}

\section{(2) OpenEdition \\ Journals}

Édition électronique

URL : https://journals.openedition.org/ahrf/144

DOI : $10.4000 /$ ahrf.144

ISSN : 1952-403X

Éditeur :

Armand Colin, Société des études robespierristes

Édition imprimée

Date de publication : 1 juin 2000

Pagination : 55-64

ISSN : 0003-4436

Référence électronique

Mariana Saad, «La médecine constitutive de la nouvelle science de l'homme : Cabanis », Annales historiques de la Révolution française [En ligne], 320 | avril-juin 2000, mis en ligne le 23 janvier 2006, consulté le 23 avril 2022. URL : http://journals.openedition.org/ahrf/144 ; DOI : https://doi.org/ 10.4000/ahrf.144

Ce document a été généré automatiquement le 23 avril 2022.

Tous droits réservés 


\title{
La médecine constitutive de la nouvelle science de l'homme : Cabanis
}

\author{
Mariana Saad
}

1 Dès l'an IV, lorsque Cabanis, nommé à l'Institut, présente à ses collègues la première vue d'ensemble de son système, la science de l'homme apparaît comme l'horizon des connaissances nouvelles qu'il va exposer. Dans le mémoire qu'il lit alors, il annonce :

«Permettez donc, Citoyens, que je vous entretienne aujourd'hui des rapports de

l'étude physique de l'homme avec celle des procédés de son intelligence; de ceux du développement systématique de ses organes avec le développement analogue de ses sentiments et de ses passions: rapports d'où il résulte clairement que la physiologie, l'analyse des idées et la morale, ne sont que les trois branches d'une seule et même science, qui peut s'appeler, à juste titre, la science de l'homme. " ${ }^{1}$

2 La construction de ce passage, qui aboutit à la formule, souligne les liens qui unissent cette science à trois différentes formes de savoirs d'une part et au couple physiquemoral d'autre part. Les membres de la classe des sciences morales et politiques sont invités à suivre un parcours que Cabanis a tracé pour eux. Il trouve son point de départ dans les rapports du physique et du moral de l'homme et aboutit à la science de l'homme ${ }^{2}$, constituée par la triade physiologie-analyse des idées-morale. Il apparait de plus, dans le dernier alinéa de ce paragraphe, que ce savoir nouveau est essentiellement médical. Cabanis le fait ainsi remarquer à son public :

«Si vous daignez me prêter quelque attention, vous verrez sans peine que le point de vue sous lequel je considère la médecine la fait rentrer à chaque instant dans le domaine des sciences morales. ${ }^{3}$

3 La proximité entre ces deux passages et le fait que l'on puisse également comprendre le couple médecine-sciences morales suivant la distinction entre le physique et le moral nous indiquent que le terme de médecine vient se substituer ici à celui de physiologie utilisé auparavant. Ceci n'est pas sans conséquences, car ainsi se trouve soulignée l'importance de la pathologie, qui est au sein de la démarche médicale et qui vient donc, par ce biais, réclamer sa place dans la science de l'homme. 
4 Mais Cabanis insiste sur l'aspect tripartite de cette dernière : dans une remarque qu'il place en note, il indique que l'expression «science de l'homme » recoupe «ce que les Allemands appellent l'Anthropologie ", et pour le démontrer, il ajoute : "sous ce titre ils comprennent en effet les trois objets principaux dont nous parlons $»^{4}$. Il n'apporte pas d'autres précisions sur l'anthropologie allemande, mais pour présenter la science de l'homme il fait appel au schéma de l'arbre de la science qu'il emprunte à Bacon. Il apparaît ainsi qu'il existe un lien essentiel entre la physiologie, l'analyse des idées et la morale. Pourtant, la distance prise avec le modèle est grande et fait surgir de nombreuses questions. Comment justifier la présence de trois sciences comme uniques branches constitutives de la science de l'homme? Quelles relations ces trois sciences entretiennent-elles à l'intérieur de ce savoir unifié que Cabanis propose?

Mais surtout, une interrogation demeure : quel rôle la médecine jouera-t-elle dans le développement de la science de l'homme?

6 Afin de répondre aux différents problèmes soulevés, il faut d'abord interroger l'idée avancée par Cabanis d'une médecine intégrée aux sciences morales. Mais pour comprendre cette affirmation, il faut la mettre en relation avec l'idée qui domine tout le mémoire : l'unité du physique et du moral. Dès lors, notre étude devra s'intéresser à la place du malade ou de la maladie dans le progrès de la science de l'homme.

7 C'est donc par une tentative de mise au jour des rapports entre la médecine et les sciences morales que nous commencerons notre démarche.

Philosophie rationnelle et science de l'homme

8 Au seuil de celle-ci, une première étape s'impose : il nous faut avancer dans la lecture du premier mémoire et nous arrêter à la manière dont Cabanis présente l'histoire de la «philosophie rationnelle ». Il affirme d'emblée :

«Sans entrer dans de grands détails, on peut voir qe les hommes qui l'ont cultivée avec le plus de succès étaient presque tous versés dans la physiologie, ou du moins que les progrès de ces deux sciences ont toujours marché de front. ${ }^{5}$

9 Cette proposition est développée tout au long du deuxième paragraphe: Cabanis s'arrête sur les périodes qui lui semblent les plus significatives et sur les grandes figures dont les travaux illustrent le mieux sa théorie. En vérité, il ne prend en considération que deux époques: l'Antiquité classique d'une part, et les Temps modernes qui s'ouvrent avec Bacon d'autre part. De l'ancienne Grèce il retient cinq " génies

extraordinaires ", «Pythagore, Démocrite, Hippocrate, Aristote et Épicure », à propos desquels il écrit :

«Tous les cinq créèrent des méthodes et des systèmes rationnels ; ils y lièrent leurs principes de morale; ils fondèrent ces principes, ces systèmes et ces méthodes sur la connaissance physique de l'homme. ${ }^{6}$

11 Ce passage apporte une grande lumière sur la nature des relations qui lient entre elles les trois branches de la science de l'homme; nous allons y revenir. Mais nous voudrions souligner dès maintenant que cette analyse s'applique à Hippocrate et qu'elle fait suite à une remarque capitale :

«[...] je mets [Hippocrate] de ce nombre, parce qu'il transporta, comme il le dit luimême, la philosophie dans la médecine, et la médecine dans la philosophie. $»^{7}$

12 Un peu plus haut, Cabanis décrit les sages grecs "occupés particulièrement de trois objets principaux»; il cite d'abord l'étude de "l'homme sain et malade», puis 
l'élaboration "des règles pour diriger leur esprit dans la recherche de vérités utiles ", enfin l'établissement des " préceptes de conduite et (de) tous les principes de morale "8.

Si nous mettons en relation ces différents extraits, nous voyons que non seulement des expressions se répètent, mais aussi qu'une correspondance s'établit terme à terme, de telle sorte que «la connaissance physique de l'homme » apparaît comme l'équivalent de l'étude de l'homme sain et malade. Il semblerait donc que physiologie et médecine se confondent ici, d'autant plus que Cabanis de nouveau emploie un mot pour l'autre quelques pages plus loin. Il est alors question de la façon dont se développe la théorie de l'entendement à l'époque moderne. Cabanis reconnaît à Bacon le mérite d'avoir inauguré cette ère nouvelle, mais c'est à Locke qu'il attribue un rôle déterminant ${ }^{9}$. Il l'explique ainsi :

«Il faut observer que Locke était médecin; et c'est par l'étude de l'homme physique qu'il avait préludé à ses découvertes dans la métaphysique, la morale et l'art social. $»^{10}$

Le glissement d'un terme à l'autre ne présente aucune difficulté. Plus encore, il suggère que la relation entre la médecine et les sciences morales repose entièrement sur le fait que le savoir médical concerne l'homme physique. La nature de cette relation reste cependant obscure. En affirmant qu'il "fait rentrer » la médecine dans les sciences morales, Cabanis paraît subordonner la première aux secondes. Mais il suppose la relation inverse lorsqu'il avance que les sages grecs se sont appuyés sur la connaissance du physique de l'homme pour élaborer une morale.

Pour lever cette confusion et éclairer du même coup le lien entre physiologie et sciences morales, il faut s'attacher à la façon dont Cabanis les conçoit. Or, la pensée de Cabanis sur cette matière est entièrement gouvernée par la conviction d'une identité entre les deux aspects du vivant. La formule est célèbre, elle se trouve dans ce même premier mémoire : «[...] le moral n'est que le physique, considéré sous certains points de vue particuliers $»^{11}$. Dans une telle perspective, connaître le physique, c'est connaître le moral et, réciproquement, l'étude du moral éclaire le physique. Pour Cabanis, les habitants de la Grèce antique ont les premiers soutenu cette idée :

"Attachés sans relâche à l'observation de la nature, les anciens remarquèrent bientôt cette correspondance de certains états physiques avec certaines tournures d'idées, avec certains penchants du caractère. ${ }^{12}$

Il passe ainsi en revue les différents tempéraments isolés alors et souligne le lien établi entre disposition du corps et état mental. Ce qui compte ici c'est la relation qui s'établit entre les différents éléments qui composent le physique et, singulièrement, les organes. Prenons un exemple, celui des hommes à «la complexation lâche et molle $»^{13}$ : leurs " qualités morales répondent à leur constitution ", elles possèdent, assure Cabanis, « un caractère de médiocrité qui, malgré l'indolence naturelle de ces individus, les rend extrêmement propores aux affaires de la vie. $»^{14}$

Comme on le voit, les médecins grecs ont établi une relation d'analogie entre le physique et le moral. Cabanis les considère, par conséquent, comme les deux aspects d'une même réalité. Plus précisément, leur développement est entièrement gouverné par celui de la sensibilité. C'est en ce sens qu'il écrit :

«[...] la vie est une suite de mouvements qui s'exécutent en vertu des impressions reçues par les différents organes; [...] les opérations de l'âme ou de l'esprit résultent aussi de mouvements exécutés par l'organe cérébral[...]. » ${ }^{15}$ 
Les impressions sont donc le fait de la sensibilité. On voit ici que les tempéraments peuvent se définir par la qualité et la quantité des sensations. Et l'importance ou la faiblesse de ces dernières trouvent leur origine dans la sensibilité, ou plutôt, dans la façon dont celle-ci s'est répartie dans le corps. Dans le deuxième mémoire, une comparaison permet de préciser l'analyse :

«[...] la sensibilité se comporte à la manière d'un fluide dont la quantité totale est déterminée, et qui, toutes les fois qu'il se jette en plus grande abondance dans un de ces canaux, diminue proportionnellement dans les autres. ${ }^{16}$

Dans l'œuvre de Cabanis, les canaux correspondent aux organes : il apparait ainsi que leur développement est proportionnel à la «quantité » de sensibilité dont ils bénéficient. Or, le cerveau, considéré comme le centre de l'intelligence et de la volonté est lui-même un organe. Cela implique que les idées et les sentiments possèdent les mêmes caractéristiques que les facultés physiques. Voilà pourquoi on trouve, dans le cinquième mémoire la remarque suivante :

«Dans les maladies de poitrine, les dispositions morales ne sont point du tout les mêmes que celles de la rate et du foie. " ${ }^{17}$

2 De cette manière, il est possible de comprendre quelle relation lie les savoirs qui forment les trois branches de la science de l'homme : la physiologie, l'analyse des idées et la morale. En effet, étudier le physique de l'homme c'est aussi découvrir de quelle manière se forment ses idées et ses penchants. Cabanis découvre à l'origine de la sagesse grecque et de l'étude de l'entendement à l'époque moderne la même conception: la connaissance du physique donne les règles de fonctionnement de l'intelligence et les lois de la morale.

Une hypothèse peut donc être avancée ici : l'ordre de la juxtaposition ne doit rien au hasard mais indique comment les savoirs sont enchaînés les uns aux autres.

Médecine, physiologie et sciences morales

Un passage de Du degré de certitude de la médecine vient d'ailleurs le confirmer: la science du physique occupe la première place dans le processus de connaissance de l'homme. Significativement, médecine est ici employé comme synonyme de physiologie :

«Elle seule (la médecine) nous fait connaître les lois de la machine vivante [...] elle nous montre à nu tout l'homme physique, dont l'homme moral n'est lui-même qu'une partie. » ${ }^{18}$

23 Telle que Cabanis la conçoit, la médecine rassemble en effet l'ensemble des savoirs sur l'homme. Ainsi, à la fin de Du degré de certitude de la médecine, il s'attarde sur la tâche du médecin et indique quelles sont les qualités nécessaires à l'exercice de cette fonction :

«Cest peu qu'il sache médicamenter; il faut qu'il sache guérir. Et pour cela, il n'a pas moins besoin de connaître les divers effets des impressions morales, que ceux des remèdes, ou des aliments. ${ }^{19}$

On le voit, il faut que le médecin puisse agir à la fois sur le corps et sur ce que Cabanis désigne dans les Rapports... d'une façon vague comme «l'esprit ou l'âme ». Or, ceci suppose que le médecin connaisse aussi bien l'homme moral que l'homme physique. Cabanis poursuit :

«Il faut qu'il soit initié dans tous les secrets du cœur, qu'il sache remuer à propos toutes les fibres sensibles. Observez les médecins qui guérissent le plus : vous verrez que ce sont presque tous des hommes habiles à manier, à tourner, en quelque sorte à leur gré, l'âme humaine. " ${ }^{20}$ 

général, ne s'intéresse à l'être humain que si celui-ci souffre, est malade. C'est donc le pathologique qui permet d'accéder à la science du normal. Une question se pose alors : pour quelle raison les caractères constants de l'être humain ne peuvent-ils être déterminés que par l'étude de ses états exceptionnels?

Le malade, la maladie et la science de l'homme toujours le résultat d'une répartition inégale de la sensibilité dans le corps. Nous avons montré ailleurs que la maladie relève pour Cabanis des catégories de l'excès et du manque : elle est avant tout dysharmonie. Elle se caractérise ainsi par la prépondérance d'un des organes sur les autres : elle met l'accent sur lui. Telle est l'analyse de Cabanis : les maladies

« [...] faisant ressortir beaucoup de phénomènes très difficiles à bien apprécier sans cela, dévoilent plusieurs ressorts, ou propriétés, qui s'effacent et disparaissent dans l'uniformité d'un état plus régulier et plus constant. » ${ }^{24}$

31 "Uniformité ", " état régulier et constant » : la santé se comprend suivant la tradition de la "crase" hippocratique, elle résulte du mélange équilibré des éléments qui constituent le corps de l'homme ${ }^{25}$. En conséquence, la maladie suppose une 
dissociation: chaque composant apparaît alors distinctement et peut être observé, étudié.

Car aux yeux de Cabanis la principale qualité d'un médecin est de savoir bien observer, de faire preuve de finesse et d'attention devant les phénomènes ${ }^{26}$. L'observation n'est pas la contemplation passive du réel : elle repose en fait sur la comparaison volontaire. Pour Cabanis, disciple de Condillac, elle est la seule source du savoir. L'homme, dit-il

«[...] se trouve jeté, comme au hasard, au milieu des scènes du monde. Les objets passent en foule sous ses yeux. C'est par leurs différences et par leurs rapports d'analogie ou de parité, qu'ils le frappent ; c'est en les comparant entre eux et avec lui, qu'il apprend à les connaître ; c'est en se comparant avec eux, qu'il apprend à se connaître lui-même. ${ }^{27}$

Dans le Traité des sensations, la statue accède aux idées de la même manière: elles naissent de la mise en rapport des différentes sensations. Et c'est ainsi que le médecin élabore son savoir : le pathologique, par les transformations qu'il fait subir aux corps, en rend certains aspects plus sensibles à l'observateur. Le malade devient un objet de connaissance à partir du moment où il devient un objet de comparaison. Le rôle qui lui est assigné est celui de «l'exote ». Nous empruntons ce terme à l'Essai sur l'exotisme de Segalen, car il nous semble le mieux à même de suggérer cette qualité d'étrangeté qui est le propre du malade et parce qu'il nous permet de mettre en rapport l'analyse de Cabanis avec un texte entièrement tourné vers les contrées lointaines: les "Considérations sur les diverses méthodes à suivre dans l'observation des peuples sauvages» de J. M. Degérando. Ce dernier justifie en effet l'intérêt scientifique de l'expédition en terres australes de Baudin de la manière suivante :

«[...] de tous les termes de comparaison que nous pouvons choisir, il n'en est point de plus curieux, de plus fécond en méditations utiles que celui que nous présentent les peuples sauvages. ${ }^{28}$

34 À l'époque de cet écrit, Degérando peut être considéré comme l'un des héritiers de Condillac $^{29}$. Mais surtout, il a lu ce mémoire au sein de la Société des Observateurs de l'Homme qui se propose de développer l'anthropologie. Or, il semble bien ici que la science de l'homme ne puisse trouver de terrain plus favorable que les peuples lointains, radicalement différents des Européens venus se comparer à eux. La position de Cabanis est toute autre : l'exotisme est au fond, pour lui, présent partout, chez tout homme et toute femme qui souffre.

Si la différence n'a pas à être cherchée au loin, c'est que le caractère essentiel de l'homme est d'être changeant. Et la médecine permet d'en prendre conscience. Les maladies peuvent s'analyser, nous l'avons vu, comme des variations de la sensibilité. Or, ces variations sont le fait des circonstances. Cabanis loue Hippocrate d'avoir su les distinguer :

"Chaque histoire forme un tableau particulier: le sexe, l'âge, le tempérament, le régime, la profession du malade y sont notés avec soin. La situation du lieu, son exposition, la nature de ses productions, les travaux de ses habitants, sa température, le temps de l'année, les changements que l'air a subis durant les saisons précédentes : telles sont les circonstances accessoires qu'il rassemble autour de ses tableaux. $»^{30}$

Dans les Rapports..., Cabanis se donne pour tâche d'étudier ces facteurs et la façon dont ils agissent sur l'homme. Il en ajoute d'ailleurs un autre, l'objet même de la recherche d'Hippocrate : la maladie. Elle modifie en effet le corps et le mouvement des idées : elle 
doit donc être prise en compte lorsqu'il ne s'agit plus d'exposer une thérapeutique mais d'élaborer la science de l'homme.

Il n'en reste pas moins vrai que c'est l'étude du pathologique qui fait apparaitre la plasticité humaine. Cabanis l'affirme :

«[...] les souffrances et les maladies sont un résultat nécessaire des lois de

l'économie animale et des circonstances au milieu desquelles l'ouvrier éternel a jeté

l'être vivant. ${ }^{31}$

L'analogie qui existe entre la forme que prend une maladie et les circonstances qui la voient éclore est la preuve de l'extrême finesse d'une sensibilité modelée par les forces qui s'exercent sur elle.

La médecine fournit ainsi à la science de l'homme une base théorique, un modèle et une démarche. Le lien avec l'Idéologie devient évident : la science de l'homme s'inscrit dans un projet à la fois épistémologique et politique: comment connaissons-nous et comment donner à l'homme les conditions les plus favorables et les plus égalitaires d'accès au savoir ${ }^{32}$.

Dans la mesure où, pour Cabanis, la médecine a pour but de contribuer au perfectionnement du genre humain, elle donne à la science de l'homme non seulement un modèle, mais aussi une dynamique.

\section{NOTES}

1.P.J.G. Cabanis, « Considérations générales sur l'étude de l'homme, et sur les rapports de son organisation physique avec ses facultés intellectuelles et morales ", premier rapport, Rapports du physique et du moral de l'homme, Genève, Slatkine reprints, 1980, p. 61.

2.L'expression « il résulte clairement » indique qu'il s'agit là d'une opération logique.

3.P.J.G. Cabanis, Rapports du physique et du moral de l'homme, op. cit., p. 61.

4.Ibid.

5.Ibid., p. 64.

6.Ibid., p. 66.

7.Ibid.

8.P.J.G. Cabanis, Rapports du physique et du moral de l'homme, op. cit., p. 64.

9.Tel est le sens de ce passage où Locke apparaît comme une véritable figure héroïque : «Depuis Bacon jusqu'à Locke, la théorie de l'entendement n'avait donc pas fait tous les progrès qu'on pouvait attendre. Mais Locke s'empare de l'axiome d'Aristote, des idées de Bacon sur le syllogisme. Il remonte à la véritable source des idées; il la trouve dans les sensations : il remonte à la véritable source des erreurs; il la trouve dans l'emploi vicieux des mots. »Ibid., p. 77.

10.Ibid.

11.Ibid., p. 78.

12.P.J.G. Cabanis, Rapports du physique et du moral de l'homme, op. cit., p. 81.

13.Ibid., p. 82. Il s'agit des pituiteux ou phlegmatiques. 
14.Ibid., p. 83.

15.Ibid., p. 78.

16.Ibid., p. 134.

17.P.J.G. Cabanis, Rapports du physique et du moral de l'homme, op. cit., p. 226.

18.P.J.G. Cabanis, Du degré de certitude de la médecine, Genève et Paris, éd. Champion-

Slatkine et éd. de la Cité des sciences et de l'industrie, 1989, pp. 8-9.

19.Ibid., pp. 152-153.

20.P.J.G. Cabanis, Rapports du physique et du moral de l'homme, op. cit., p. 153.

21.Les limites de cet article ne nous permettant pas d'aborder cette question, nous renvoyons le lecteur aux chapitres II et III de notre thèse, Santé et maladie dans l'œuvre de P.J.G. Cabanis, Université de Paris I, 1997.

22.P.J.G. Cabanis, Du degré de certitude de la médecine, op. cit., p. 9.

23.Ibid.

24.P.J.G. Cabanis, Révolutions et réforme de la médecine, Euvres complètes, tome I, Paris, 1823, p.19.

25.Hippocrate, Nature de l'homme, c4.

26.P.J.G. Cabanis, Rapports du physique et du moral de l'homme, op. cit., p. 89.

27.P.J.G. Cabanis, Du degré de certitude de la médecine, op. cit., p. 80.

28.Joseph-Marie Degérando, «Considérations sur les diverses méthodes à suivre dans l'observation des peuples sauvages ", in J. Copans et J. Jamin, Aux origines de l'anthropologie française, Paris, Le Sycomore, 1978, p. 130.

29.Dans sa thèse, La Société des Observateurs de l'homme (1799-1804), genèse, personnel et activités d'une société savante sous le Consulat, (Université de Paris I, 1999), J.-L. Chappey décrit avec soin les différentes positions intellectuelles défendues par Degérando au cours de sa vie.

30.P.J.G. Cabanis, Rapports du physique et du moral de l'homme, op. cit., p. 71.

31.P.J.G. Cabanis, Du degré de certitude de la médecine, op. cit., p. 14.

32.Sur le lien entre ces deux ambitions de l'Idéologie, voir Pierre Macherey, «L'Idéologie avant l'Idéologie : l'École normale de l'an III », in L'institution de la raison : la Révolution culturelle des Idéologues, sous la direction de Fr. Azouvi, Paris, Vrin, Ehess, 1992.

\section{RÉSUMÉS}

Dès le premier mémoire des Rapports du physique et du moral de l'homme - son œuvre somme Cabanis désigne la comme le point d'aboutissement de sa réflexion. Il indique que cette science est semblable à un arbre dont les branches seraient la physiologie, l'analyse des idées et la morale. En étudiant la manière dont Cabanis envisage ces différentes formes de savoir, il apparaît que la médecine - synonyme de physiologie - est non seulement la porte d'accès privilégiée à la connaissance de l'homme, mais qu'elle est aussi l'autre nom de la science de l'homme. Pour comprendre ceci il faut, d'une part, se reporter à la thèse centrale des Rapports... qui pose l'identité du physique et du moral et, d'autre part, à la manière dont Cabanis conçoit l'homme, 
être plastique dont la maladie fait ressortir des aspects particuliers, permettant par là de les observer et de les étudier.

\section{Medicine as a constituent of the new Science of the Man : Cabanis}

In his first treatise and magnum opus, the Rapports du physique et du moral de l'homme, P. J. G. Cabanis identifies the «science of man» as the final outcome of his thinking. He points out that this science resembles a tree whose branches are physiology, the analysis of ideas and morality. A study of the manner in which Cabanis envisioned these different branches of knowledge reveals that medecine - synonimous with physiology - is not only the portal through which an understanding of man is possible, but also another name for the science of man. To understand this, it is necessary to refer on the one hand to the central thesis of the Rapports which posits the identity of the physical and the moral, and on the other, to the way Cabanis conceives of man, as

a plastic being whose specific features emerge through disease and can then be observed and investigated.

INDEX

Mots-clés : Cabanis, médecine, physiologie, science de l'homme, sciences morales et politiques

\section{AUTEUR}

MARIANA SAAD

Docteur en philosophie. Université de Paris I - Panthéon-Sorbonne 